\title{
HPV18 E6 and E7 Intratumour Heterogeneity in Esophageal Cancer
}

\author{
Sara Khodahemmati ${ }^{1 \#, ~ M a l i h a ~ G a f f a r ~}{ }^{1 \#}$, Jintao Li $^{1 *}$, Yangjunqi Wang ${ }^{1}$, Xiaoli Wang1, \\ Zhixiang Zhou', Yi Zeng1,2*
}

\footnotetext{
${ }^{1}$ Beijing Key Laboratory of Environmental and Viral Oncology, College of Life Science and Bio-Engineering, Beijing University of Technology, Beijing, China

${ }^{2}$ State Key Laboratory of Infectious Disease Prevention and Control, National Institute for Viral Disease Control and Prevention, Chinese Center for Disease Control and Prevention, Collaborative Innovation Center for Diagnosis and Treatment of Infectious Diseases, Beijing, China

Email: *zengyicd@@sina.com, ${ }^{\star}$ Ljt2008@bjut.edu.cn
}

How to cite this paper: Khodahemmati, S., Gaffar, M., Li, J.T., Wang, Y.J.Q., Wang, X.L., Zhou, Z.X. and Zeng, Y. (2019) HPV18 E6 and E7 Intratumour Heterogeneity in Esophageal Cancer. Journal of Cancer Therapy, 10, 352-360.

https://doi.org/10.4236/jct.2019.105029

Received: November 7, 2018

Accepted: May 6, 2019

Published: May 9, 2019

Copyright $\odot 2019$ by author(s) and Scientific Research Publishing Inc. This work is licensed under the Creative Commons Attribution International License (CC BY 4.0).

http://creativecommons.org/licenses/by/4.0/

\section{(c) (i) Open Access}

\begin{abstract}
The development of esophageal cancer accompanied by the presence of human papillomavirus (HPV) DNA into the host genome. By evaluating the expression of this virus for tumor cell origin and also their cell grows and migrations, we examined esophageal cancer clonality in the context of intra-tumor heterogeneity. In this research, we have checked the expression of HPV18 E6 and E7 in different single cell clones by the manual cell picking method in the HPV positive esophageal cancer (EC109), EC109 cell line used as a negative control, and Hela cell line used as the positive control. Quantitative real-time PCR (QRT-PCR) was run to detect the expression levels of HPV E6 and E7, Cell Counting Kit-8 (CCK-8) assay was used to examine cell proliferation, invasion assays performed using Costar chambers and wounding assay to study cell migrations in vitro. We investigated the intra-tumor heterogeneity of HPV E6 and E7 in esophageal cancer and the evaluation of the growth and migrations at the clonal level, using 10 single cell clones. In particular clones, C7 \& C10 displayed a highly variable expression in both HPV E6 and E7 and weak in four clones (C1, C3, C4, and C9) consequently, the cell invasion, proliferation, and migration increase with increasing the level of HPV expression and inverse. In conclusion, the resulting based on single cell cloning showed the relationship between HPV and cell growth and migration in esophageal cancer. Future study in HPV DNA integration needed to explore the mains specific integration site of HPV DNA in esophageal cancer and molecular monitoring of the HPV for future prevention researches and also effective therapeutic strategies.
\end{abstract}




\section{Keywords}

Esophageal Cancer, Human Papillomavirus, HPV 18 E6 and E7, Single Cell

Cloning, Intra-Tumor Heterogeneity

\section{Introduction}

The population of cancer cells have detected in different researches and explained heterogeneity in term of tumorigenicity, mutations, activation of metabolic and signaling pathway, grows and migrations, different ploidy, metastasis, alternation of copy number, and responding to the anticancer agent [1] [2] [3]. Cell heterogeneity can be observed in the different group of patients and also different tumors in the same organ (inter-tumor heterogeneity) and different cells in the same tumor (intra-heterogeneity) [4] [5] [6].

The idea of the intera-heterogeneity is back to at least to the 1970s when the same tumor in the mouse models had different sensitivities to cytotoxic therapy or different tendencies to metastasize [7].

Tumor heterogeneity has observed in different kind of cancers like esophageal cell carcinoma [8]. Afterward, studies detected the HPV DNA in esophageal cancer base on intra-heterogeneity. Research evidence that, the integration of HPV DNA may result in esophageal cancer intra-heterogeneity. Esophageal cancer is following to steady infection of esophagus epithelial cells with oncogenic types of HPVs [9]. Early carcinogenesis described by constant integration of HPV DNA into the host genome [10]. Following, rising HPV DNA integrity results in increasing HPV DNA expressions and consequently, cell grows and proliferation [11].

However, the HPV DNA contribution in the context of single clones has not widely studied. In this research we are going to proof intra-tumour heterogeneity in esophageal cancer by detecting HPV E6 and E7 genes in 10 different single clones of esophageal cancer cells and clonal expansion by evaluating proliferation as well as invasions and migrations of each single clone to better understand HPV E6 and E7 roles in ESCC intra-heterogeneity and disease monitoring.

\section{Materials and Methods}

\subsection{Cell Lines and Single Cell Clones}

The esophageal squamous cell carcinoma cell lines (EC109) were obtained from the Zeng Academician Laboratory of the Virus Prevention and Control Institute (CDC, Beijing, China). All cells were cultured in RPMI-1640 medium (Hyclone, USA) supplemented with $10 \%$ fetal bovine serum (10\% FBS), and they were seeded in 96-well plates at a calculated 1 cell/well in each test medium, and maintained in a humidified incubator at $37^{\circ} \mathrm{C}$ with $5 \% \mathrm{CO}_{2}$.

\subsection{RNA Extraction and $q R T-P C R$}

We used Trizol reagent (Takara, Japan) to isolate the Total RNA from culture's 
cell, and then using the Prime Script RT reagent Kit (Promega, USA) to synthesize the cDNA from a total of $400 \mathrm{ng}$ RNA according to the manufacturer's protocol. cDNA amplified by quantitative real-time PCR with SYBR Green Kit (Promega, USA). For normalizing the level of HPV18 E6 \& E7 we used GAPDH. Primers for HPV 18E6, E7 and GAPDH are listed in Additional Table 1.

\subsection{Cell Proliferation Assay}

Cell proliferation was performed every 12 hours in 96-well plates and 1000 cells per wells by using cell counting kit8 (Djingo, Japan) as the manufacturer's protocol.

\subsection{Invasion Assays}

Single clone cells seeded $\left(5 \times 10^{4}\right)$ were suspended in $200 \mu \mathrm{l}$ of serum-free medium and seeded in the upper Costar chambers containing transwell inserts with a pore size of $8 \mu \mathrm{m}$ (Corning Incorporated, USA), and coated with Matrigel (Invitrogen, USA). While the bottom chamber contained medium mixed with $20 \%$ FBS. We were fixed the cells with methanol and stained with $0.1 \%$ crystal violet, then removing the extra cells by using cotton swap in the upper chamber after forty-two hours. Then imaged, and counted under an inverted microscope in three random fields (Olympus, Japan).

\subsection{Wounding Assay}

The wound was made on the monolayer cells and capturing. Cells started to migrate at the wound edges then we have captured the images as the second stages of our experiment after 12 hours. Then we have calculated the migrations speed of the cells in certain clones by comparing these two stages together.

\subsection{Statistical Analysis}

The statistical significance analysis was examined at the mean \pm standard deviation (SD), using T-test and ANOVA. All analyses were performed on SPSS 21.0, and $(P<0.05)$ was considered significant.

\section{Results}

\subsection{Esophageal Cancer Single Clones and RT-PCR Analysis of HPV E6 and E7 Expression}

To analyze the expression of HPV18 E6 and E7 at the clonal level, 10 single cell clones were obtained from a primary culture of the human esophageal cancer (EC109). In this case, we used manual cell picking metode. The cells are typically provided as a suspension in a 96 well-plate. The single-seeded cell was choosing via microscope observation. And obtained clones from these single cells were cultured in RPMI-1640 medium (Hyclone, USA) supplemented with $10 \%$ fetal bovine serum $(10 \% \mathrm{FBS})$, and maintained in a humidified incubator at $37^{\circ} \mathrm{C}$ with5\% $\mathrm{CO}_{2}$. These clones were evaluated by the Qrt-PCR to detect the expression level of 
Table 1. Primers for HPV E6 and E718 E6, E7 and GAPDH

\begin{tabular}{ccc}
\hline & SENSE(5'-3') & ANTISENSE(5'-3') \\
\hline GAPDH & ACCACAgTCCATgCCATCAC & TCCACCACCCTgTTgCTgTA \\
E718 E6 & ggTgCCAgAAACCgTTgA & TgCgTCgTTggAgTCgT \\
HPV18 E7 & TAAgCgACTCAgAggAAgAA & gCTggAATgCTCgAAgg \\
\hline
\end{tabular}

HPV18 E6 and E7 fragments. Results revealed that a heterogeneous expression of HPV E6 and E7 in investigated clones (Figure 1) and significant difference between them $(\mathrm{P}<0.05)$. According to these results, particular clones displayed a highly variable expression in both HPV E6 and E7 (C7, C8 and C10) had low expression in clones $\mathrm{C} 3, \mathrm{C} 4$, and $\mathrm{C} 5$.

\subsection{Cell proliferation in Different Single Clones}

We were used CCK-8 assays to detect the effect of intra-heterogeneity on cell proliferation and growth as shown in (Figure 2). Significant difference was observed between clones in proliferation $(\mathrm{P}<0.05)$. Our results demonstrated those clones which have high expression of HPV 18 E6 and E7 significantly promoted cell proliferation.

\subsection{Single Clone Cells Migration and Invasion}

To detect the functions of HPV18 E6 and E7 in esophageal cancer migration and invasion, we conduct the wounding and Tran swell chamber assays. The significant difference was observed between clones in both wounding and invasion assays $(\mathrm{P}<0.001)$. Single clones which had high expression of HPV 18 E6 and E7 significantly promoted cell migration through a permeable filter and invasion through Matrigel Matrix (Figure 3 and Figure 4).

\section{Discussions}

One of the main reasons for poor effective therapies in esophageal cancer is an inappropriate prognosis [12]. Study on cancer cell heterogeneity is required for detecting the appropriate molecular prognostic markers as well as patients' classifications and using them for specific and effective therapies. Afterward, research efforts for heterogeneity descriptions would be helpful for better understanding of the disease progressing and diagnosis [11].

Heterogeneity mentioned the different morphology and genotype in distinct tumor cells, this phenomenon can happen between tumors and also within tumors which is called inter-tumor heterogeneity and intra-tumor heterogeneity respectively [4] and caused by both genetic and non-genetic factors in a variety of cancers as well as esophageal cancer [13].

HPV E6 and E7 in esophageal cancer have detected [14], and the role of these oncogenes in heterogeneity in plenty of researches evaluated. 


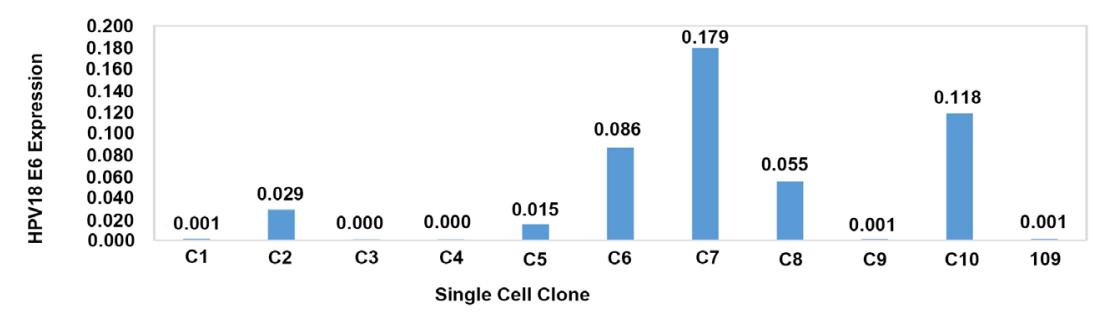

(a)

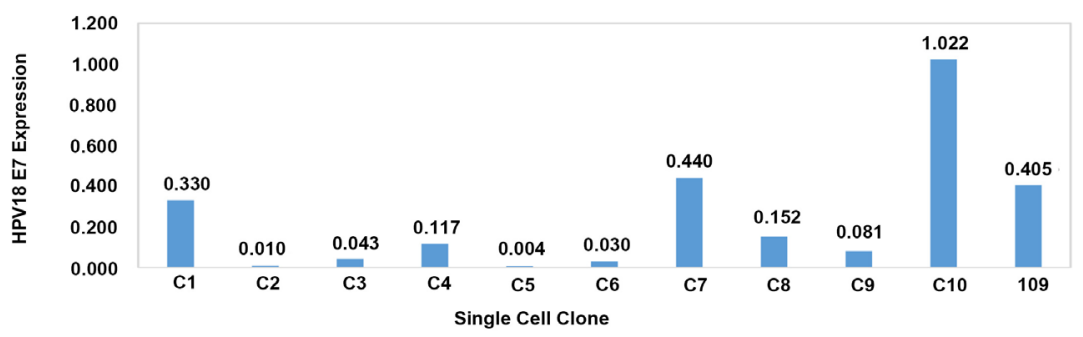

(b)

Figure 1. QRT-PCR analysis performed to evaluate the expression of HPV 18 E6 (a) and E7 (b) in 10 single clones of HPV positive esophageal cancer (EC109), EC109 as a negative control and Hela cell line used as the positive control. Significantly difference observed between different clones $(\mathrm{P}<0.05)$.

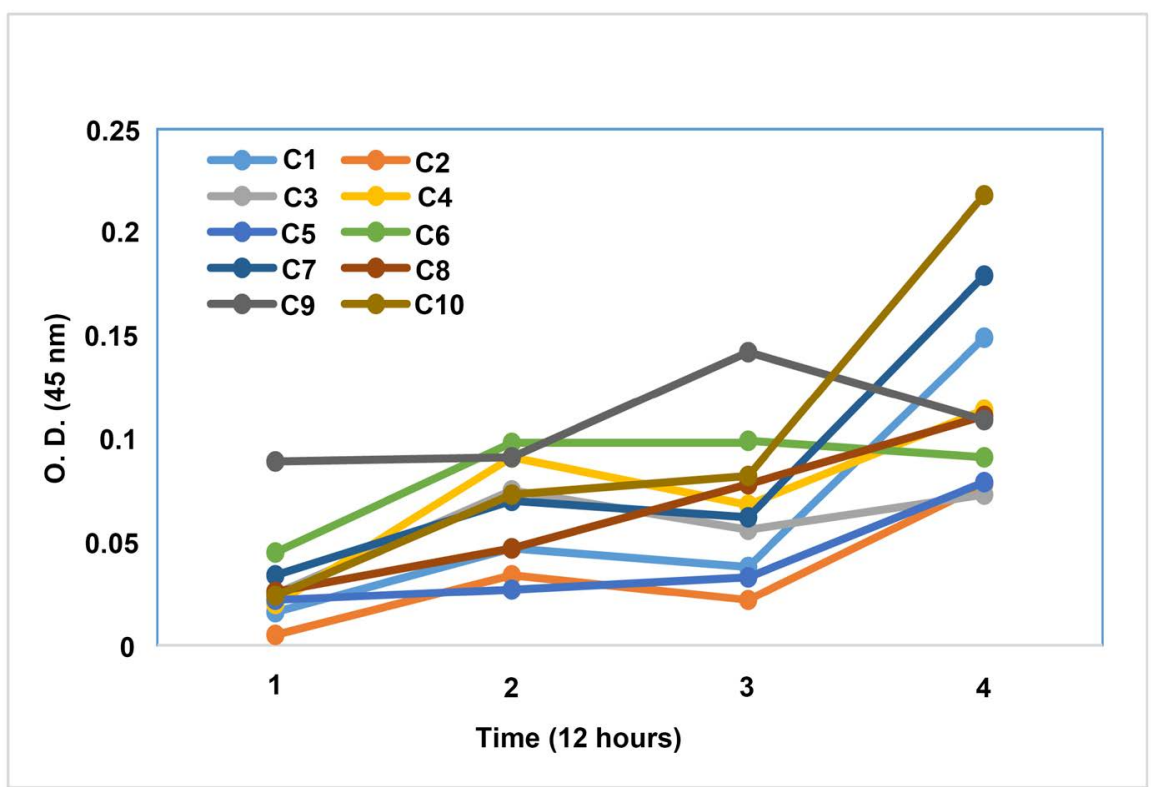

Figure 2. The proliferation assay performed, using CCK-8 kit every 12 hours and results of 10 single Cell clones and EC109 celline, considering a significant difference between clones $(\mathrm{P}<0.05)$.

The studies demonstrated that the different HPV gene expressions in each single clones. It is may because of HPV DNA can exist in tow form in human genome, integrate or non integrated. Upon infection, firstly HPV genome amplified as episomes in the cell then some of them subsequently integrate randomly in to the host genome in one or more different location. Research showed 


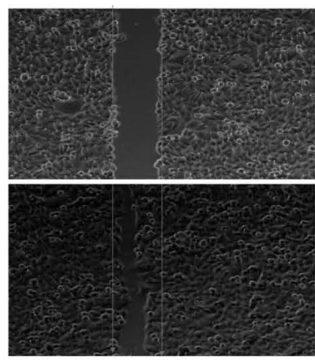

c1

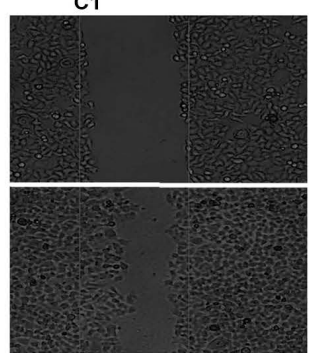

c6
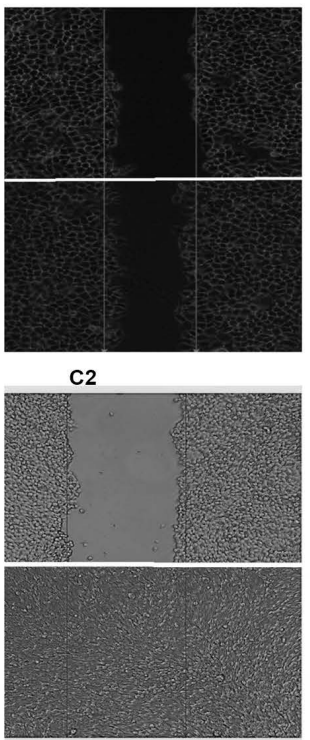

C7

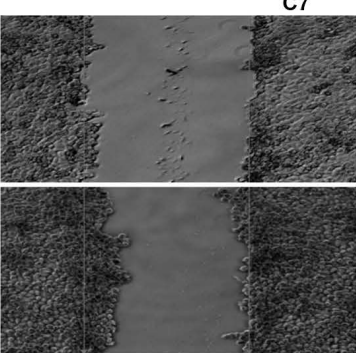

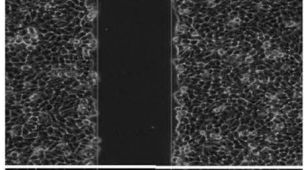

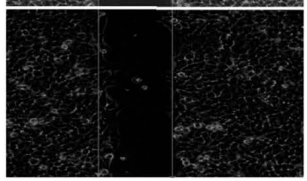

C3

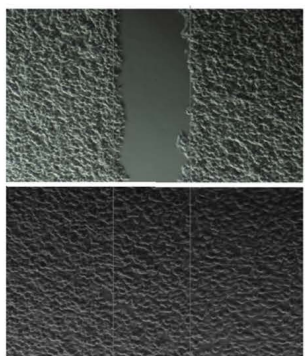

C8

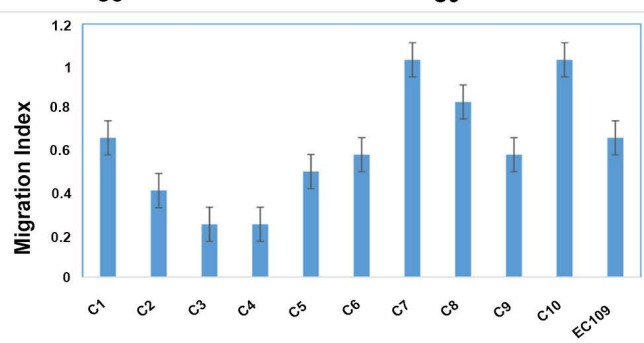

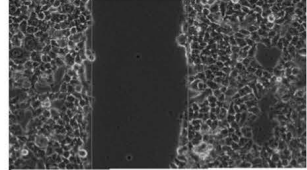

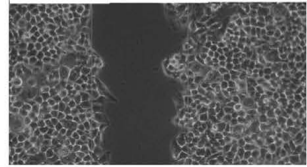

C4
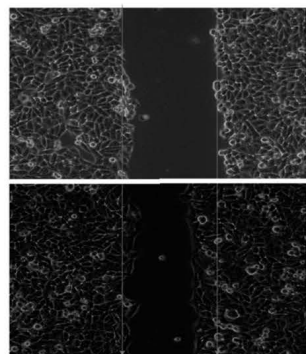

C9

C10

C5
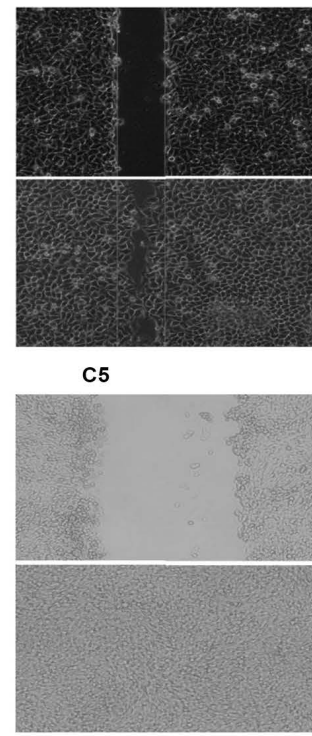

Figure 3. Wound healing assay performed in 10 different single clones of EC109 cellines. the wounds were captured pictures at 0 and 12 hours post-scratch $(10 \times$ magnifications) and the results calculated at the two time points in different single clones as well as control cell lines. Significantly difference was observed between cell lines $(\mathrm{P}<0.001)$.
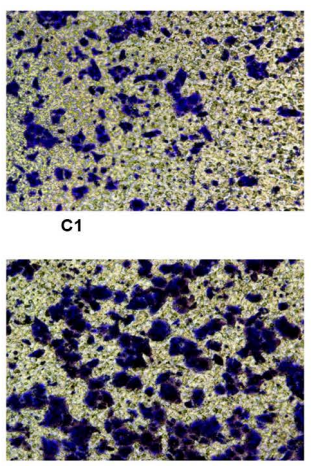

c6

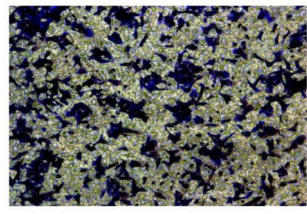

c2

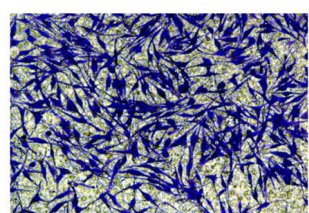

c7

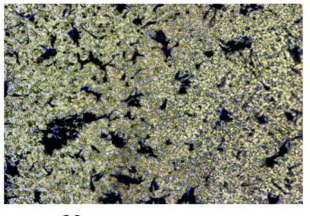

c3

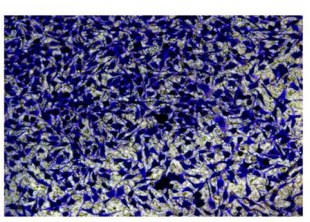

C8

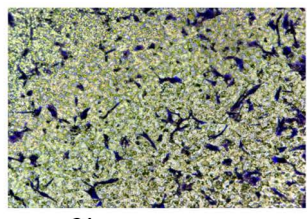

C4

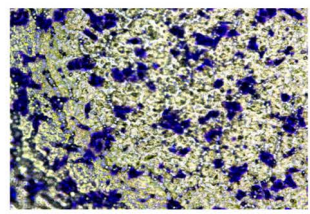

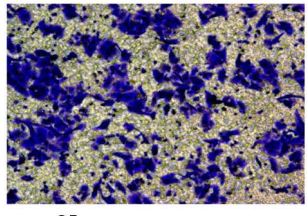

C5

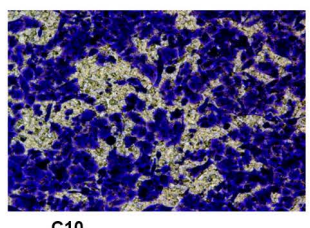

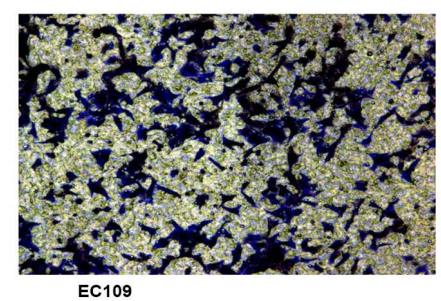

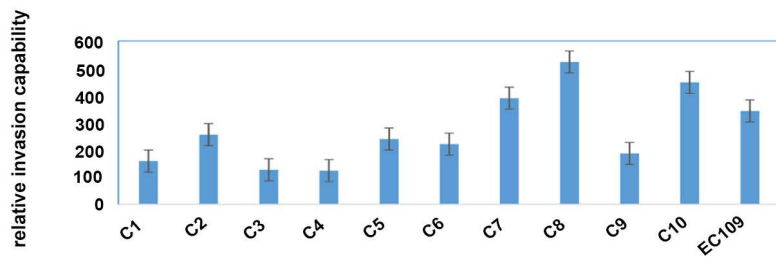

Figure 4. Invasion assay performed in 10 single clones of EC109 cell line, EC109 and Hela used as a negative and positive control controls, using a transwell system. And the cells were captured in 10x magnifications. Significantly invasion observed between different single clones $(\mathrm{P}<0.001)$. 
that there is strongly associated between HPV integrate DNA and proliferation increasing in compare with episomal HPV DNA [11].

In this study 10 single clone HPV 18 positive esophageal cancers were obtained and the expression levels of E6 and E7 oncogenes were detected indifferent single clones. The results showed the heterogeneous expression of E6 and E7. Some clones explaned highly expressed of both E6 and E7 (7C, C8 and C10) and some had low expression of these tow oncogenes (C3, C4 and C5). As far as Human papillomavirus $16 / 18$ promotes cancer cell proliferation, migration and invasion [15], this study was conducted to check the cell cycle regulations of each single clone and result demonstrate that those single clone cells with high expression of HPV E6 and E7 had high level of proliferation, migrations and also invasion.

\section{Conclusion}

This research revealed that the role of HPV 18 E6 and E7 in cell regulations in the context of intra-heterogeneity in ESCC. It may potentially help to monitor and progress of HPV positive esophageal cancer therapies and diagnostic.

\section{Acknowledgements}

This study was supported by Beijing Natural Science Foundation, Beijing University of Technology Foundation, Development Program of China and National Key Technology Support Program.

\section{Funding}

This study was supported by Beijing Natural Science Foundation (Grant No. 5162003); Beijing University of Technology Foundations (Grant No. 015000514314004); Development Program of China (Grant No. 2011SLKID103); National Key Technology Support Program (Grant No. 2006BAI19B03).

\section{Availability of Data and Materials}

All available data can be obtained by contacting the corresponding author.

\section{Ethical Approval}

All procedures performed in studies involving human participants were in accordance with the ethical standards of the institutional and national research committee and with the 1964 Helsinki declaration and its later amendments or comparable ethical standards.

\section{Informed Consent}

Informed consent was obtained from all individual participants included in the study.

\section{Patient Consent for Publication}

Patient consent was obtained from all individuals in this study. 


\section{Conflicts of Interest}

The authors declare no conflicts of interest regarding the publication of this paper.

\section{Manuscript Materials}

The manuscript contains original material. And the content has not been published or submitted for publication elsewhere.

\section{References}

[1] Doorbar, J. (2006) Molecular Biology of Human Papillomavirus Infection and Cervical Cancer. Clinical Science, 110, 525-541. https://doi.org/10.1042/CS20050369

[2] Somasundaram, R., Villanueva, J. and Herlyn, M. (2012) Intratumoral Heterogeneity as a Therapy Resistance Mechanism: Role of Melanoma Subpopulations. Advances in Pharmacology, 65, 335-359. https://doi.org/10.1016/B978-0-12-397927-8.00011-7

[3] Carow, K., Gölitz, M., Wolf, M., Häfner, N., Jansen, L., Hoyer, H., et al. (2017) Viral-Cellular DNA Junctions as Molecular Markers for Assessing Intra-Tumor Heterogeneity in Cervical Cancer and for the Detection of Circulating Tumor DNA. International Journal of Molecular Sciences, 18, Article No. 2032. https://doi.org/10.3390/ijms18102032

[4] Tellez-Gabriel, M., Ory, B., Lamoureux, F., Heymann, M.F. and Heymann, D. (2016) Tumour Heterogeneity: The Key Advantages of Single-Cell Analysis. International Journal of Molecular Sciences, 17, Article No. 2142. https://doi.org/10.3390/ijms17122142

[5] Michor, F. and Polyak, K. (2010) The Origins and Implications of Intratumor Heterogeneity. Cancer Prevention Research, 3, 1361-1364. https://doi.org/10.1158/1940-6207.CAPR-10-0234

[6] Visvader, J.E. (2011) Cells of Origin in Cancer. Nature, 469, 314-322. https://doi.org/10.1038/nature09781

[7] Mroz, E.A. and Rocco, J.W. (2016) Intra-Tumor Heterogeneity in Head and Neck Cancer and Its Clinical Implications. World Journal of Otorhinolaryngology-Head and Neck Surgery, 2, 60-67. https://doi.org/10.1016/j.wjorl.2016.05.007

[8] Wen, D., Zhang, L., Wang, X., Wen, X., Yang, Y., Chen, Y., et al. (2017) Heterogeneity in Esophageal and Gastric Cardia Precursor Progression during Six-Year Endoscopic Surveillance after Population-Based Screening in a Chinese High-Risk Region. Thoracic Cancer, 8, 328-336. https://doi.org/10.1111/1759-7714.12446

[9] Hausen, H.Z. (2002) Papillomaviruses and Cancer: From Basic Studies to Clinical Application. Nature Reviews Cancer, 2, 342-350. https://doi.org/10.1038/nrc798

[10] Jeon, S., Allen-Hoffmann, B.L. and Lambert, P.F. (1995) Integration of Human Papillomavirus Type 16 into the Human Genome Correlates with a Selective Growth Advantage of Cells. Journal of Virology, 69, 2989-2997.

[11] Beck, T.N. and Golemis, E.A. (2016) Genomic Insights into Head and Neck Cancer. Cancers of the Head and Neck, 1, Article ID: 6423.

https://doi.org/10.1186/s41199-016-0003-Z

[12] Hao, J.J., Lin, D.C., Dinh, H.Q., Mayakonda, A., Jiang, Y.Y., Chang, C., et al. (2016) Spatial Intratumor Heterogeneity of Genetic, Epigenetic Alterations and Temporal Clonal Evolution in Esophageal Squamous Cell Carcinoma. Nature Genetics, 48, 
1500-1507. https://doi.org/10.1038/ng.3683

[13] Walter, D. (2017) Intratumoral Heterogeneity of Esophageal Squamous Cell Carcinoma. Journal of Thoracic Disease, 9, 465-467. https://doi.org/10.21037/jtd.2017.04.54

[14] Zhao, X., Sahi, R., Zhao, Y.Y., Wang, J. and Li, C.H. (2015) Expressions of HPV 16-E6 in Esophageal Carcinoma and It's Clinical Significance. Asian Journal of Medical Sciences, 6, 36-42. https://doi.org/10.3126/ajms.v6i6.12537

[15] Liao, S., Deng, D., Zhang, W., Hu, X., Wang, W., Wang, H., Lu, Y., Wang, S., Meng, L. and Ma, D. (2102) Human Papillomavirus 16/18 E5 Promotes Cervical Cancer Cell Proliferation, Migration and Invasion in Vitro and Accelerates Tumor Growth in Vivo. Oncology Reports, 29, 95-102. https://doi.org/10.3892/or.2012.2106 